\title{
LES on Plume Dispersion in the Convective Boundary Layer Capped by a Temperature Inversion*
}

\author{
Hiromasa NAKAYAMA $^{* *}$, Tetsuro TAMURA ${ }^{* * *}$ and Satoshi ABE ${ }^{* * *}$ \\ **Japan Atomic Energy Agency,Tokai-mura, Naka-gun, Ibaraki \\ E-mail: nakayama.hiromasa@jaea.go.jp \\ *** Tokyo Institute of Technology, \\ Nagatsuta4259, Midori-ku, Yokohama \\ E-mail: tamura@depe.titech.ac.jp
}

\begin{abstract}
Large-eddy simulation (LES) is applied to the problem of plume dispersion in the spatially-developing convective boundary layer (CBL) capped by a temperature inversion. In order to generate inflow turbulence with buoyant forcing, we first, simulate the neutral boundary layer flow (NBL) in the driver region using Lund's method. At the same time, the temperature profile possessing the inversion part is imposed at the entrance of the driver region and the temperature field is calculated as a passive scalar. Next, the buoyancy effect is introduced into the flow field in the main region. We evaluate the applicability of the LES model for atmospheric dispersion in the CBL flow and compare the characteristics of plume dispersion in the CBL flow with those in the neutral boundary layer. The Richardson number based on the temperature increment across the inversion obtained by the present LES model is 22.4 and the capping effect of the temperature inversion can be captured qualitatively in the upper portion of the CBL. Characteristics of flow and temperature fields in the main portion of CBL flow are similar to those of previous experiments ${ }^{[1],[2]}$ and observations ${ }^{[3]}$. Concerning dispersion behavior, we also find that mean concentrations decrease immediately above the inversion height and the peak values of r.m.s concentrations are located near the inversion height at larger distances from the point source.
\end{abstract}

Key words: LES, Convective Boundary Layer, Temperature Inversion, Dispersion

\section{Introduction}

Atmospheric dispersion of toxic and flammable gases resulting from accidental or intentional releases is of great concern to public health. For safety analysis, it is therefore especially important to investigate the fluctuating characteristics of a plume. Many wind tunnel experiments have been conducted on concentration fluctuation of plume dispersion in a turbulent boundary layer ${ }^{[4],[5],[6]}$, etc. For example, Fackrell et al ${ }^{[4]}$ investigated the characteristics of concentration fluctuation of gases emitted from the ground and elevated sources in a turbulent boundary layer. However, these studies have focused on the characteristics of plume dispersion under neutral conditions. Heating and cooling within the boundary layer during the daily solar cycle result in temperature differences, which introduce buoyant forcing. Buoyancy effects on plume dispersion are important factors in clarifying the mechanism of atmospheric dispersion under more realistic conditions. 
Therefore, in recent studies of atmospheric dispersion in thermally stratified environments, for example, Sada ${ }^{[7],[8]}$ analyzed the similarity of flow and dispersion fields between wind tunnel and atmospheric flows. He found that the maximum ground level concentration increased and occurred closer to the source in wind tunnel experiments on gas dispersion in the convective boundary layer $(\mathrm{CBL})$. Fedorovich et al. ${ }^{[1]}$ conducted a wind tunnel experiment on plume dispersion in the convective boundary layer capped by a temperature inversion and investigated the influence of relative source elevation to the capping inversion height on the longitudinal distribution patterns of concentration.

Our objectives are: (a) to propose a numerical model for LES (Large Eddy Simulation) on atmospheric dispersion in the spatially-developing CBL flow capped by a temperature inversion, (b) to compare plume behaviors in the CBL flow and the NBL flow, and (c) to investigate the capping effects of temperature inversion on the characteristics of mean and fluctuating concentrations.

\section{Nomenclature}

c: concentration

$\mathrm{c}_{\text {mean }}$ : mean concentration

$\mathrm{c}_{\text {mean_max }}$ : maximum mean concentration

$\mathrm{c}_{\mathrm{rms}}$ : r.m.s. of fluctuating concentration

Crms_max: maximum r.m.s concentration

$\mathrm{C}_{\mathrm{o}}$ : source concentration

g: gravity acceleration

Hs: source height

p: pressure

t: time

$\mathrm{u}_{\mathrm{i}}$ : wind velocity

$\mathrm{u}_{*}$ : friction velocity

$\mathrm{U}_{\mathrm{e}}$ : velocity of effluent

$\mathrm{U}_{\mathrm{m}}$ : wind velocity which remains constant with height inside the CBL

$\mathrm{U}_{\text {mean }}$ : mean velocity

$\mathrm{U}_{\infty}$ : free stream velocity

$\mathrm{u}_{\mathrm{rms}}, \mathrm{v}_{\mathrm{rms}}, \mathrm{w}_{\mathrm{rms}}$ : turbulent intensity

u' $\theta$ ', w' $\theta$ ': streamwise, vertical heat fluxes

$\mathrm{w}^{2}$ : vertical velocity variances

$\mathrm{W} *$ : convective velocity scale

w'c': vertical concentration flux

$\mathrm{x}, \mathrm{y}, \mathrm{z}$ : coordinates

$\mathrm{z}_{\mathrm{i}}$ : inversion height

$\tau_{i j}$ : subgrid-scale Reynolds stress

$h_{j}$ : subgrid-scale heat flux

$s_{j}$ : subgrid-scale concentration flux

Pr: Prandtl number

Re: Reynolds number

Sc: Schmidt number

$\mathrm{Sc}_{\text {sgs }}$ : turbulent Schmidt number

$\beta$ : thermal expansion coefficient

$\theta:$ temperature

$\theta^{2}:$ temperature variances

$\theta_{*}$ : convective temperature scale

$\theta m$ : temperature which remains constant with height inside the $\mathrm{CBL}$

$\theta_{0}:$ reference temperature

$\theta_{s}:$ surface temperature

$\theta_{t}$ : averaged absolute temperatures within the inversion layer

$\triangle \theta_{t}$ : temperature increment across the inversion

$\delta$ : thickness of NBL at inlet of main region 
$\delta_{\mathrm{o}}$ : thickness of NBL at inlet of driver region

$v$ :kinematic viscosity

$\rho$ : density

\section{Numerical Model}

\subsection{Computational Model for CBL Flow Capped by a Temperature Inversion}

So far, various kinds of the methods for producing a realistic turbulent boundary layer are proposed. For example, Spalart et al. ${ }^{[9]}$ propose the fringe method. An artificial damping term is introduced to the equation of motion in a region called fringe region by imposing the periodic boundary condition in the streamwise direction. However, the effects on turbulent flow field have not been discussed by introducing this method. Maeder et al. ${ }^{[10]}$ propose the extended temporal method. Turbulent flow in the computational domain is calculated at a series of downstream positions by using the periodic boundary condition. Therefore, turbulent boundary layer flow can be obtained in much shorter domain size (1 to 5 times of boundary layer thickness. However, it is difficult to capture the steady structure of turbulent boundary layer. On the other hand, Lund et al. ${ }^{[11]}$ proposed the rescaling method for simulating a spatially-developing boundary layer using quasi-periodic boundary conditions. Turbulent flow in the specific positions can be easily obtained in comparatively shorter domain size (10 times of turbulent boundary thickness). In this study, we adopt this method and extend it to simulation of a spatially-developing CBL flow capped by a temperature inversion $^{[12]}$.

Figure 2.1 shows a numerical model for atmospheric dispersion in a spatially-developing CBL flow capped by a temperature inversion. Firstly, we carry out LES on a fully-developed neutral boundary layer flow using Lund's method ${ }^{[11]}$. At the same time, we calculate the temperature field as passive scalar in the driver region. At the inlet of the driver region, the temperature profile possessing the inversion part is regulated as shown in Fig. 2.2. Here, $z_{i}$ is the inversion height defined as the elevation of the heat flux minimum within the inversion layer. $\theta m$ and $\theta s$ are the temperature that remains constant with height inside the CBL and the surface temperature, respectively. Next, the inflow turbulence and temperature data generated in the driver region are imposed at the entrance of the main computational region. Here, we incorporate buoyancy effects modeled by Boussinesq approximation into this main computational region and perform a numerical simulation of a spatially-developing flow. A tracer gas is emitted from a point source located away from the entrance of the main computational region, and LES on plume dispersion in the simulated spatially-developing CBL flow is carried out.

Figure 2.3 shows the computational model. The sizes of the computational region are $40 \delta_{\mathrm{o}}$ $\times 6.4 \delta_{0} \times 3.0 \delta_{0}\left(\delta_{0}\right.$ : the thickness of NBL at the inlet of driver region) in the $\mathrm{x}-, \mathrm{y}-$, $\mathrm{z}$-directions, respectively. Here, $\mathrm{x}, \mathrm{y}$ and $\mathrm{z}$ indicate streamwise, spanwise and vertical directions, respectively. The recycle station is located $8.3 \delta_{\text {o }}$ downstream of the inlet in the driver region. The velocity field is rescaled at the recycle station and re-introduced at the

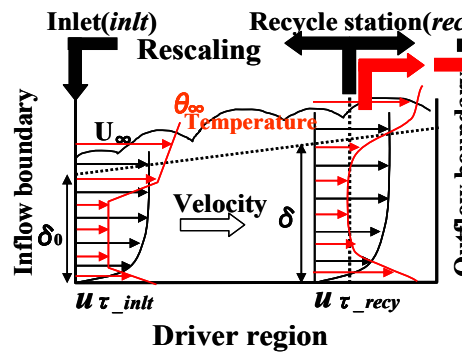

Fig. 2.1 Numerical model for plume dispersion in a spatially-developing CBL flow capped by a temperature inversion
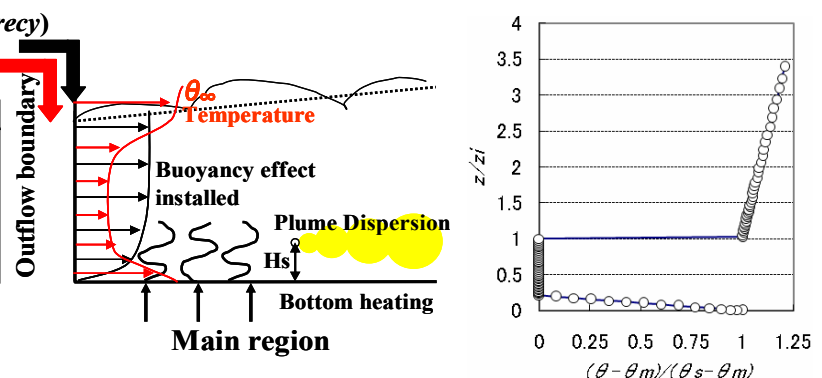

Fig. 2.2 Temperature profile regulated at the inlet of driver region 
inlet. The turbulent inflow generated at the recycle station is imposed at the inlet of the main computational region. The grid number of the computational region is $400 \times 128 \times 90$ in the $\mathrm{x}-, \mathrm{y}-, \mathrm{z}$-directions, respectively. The grid resolution is $\Delta \mathrm{x}^{+}=22.0, \Delta \mathrm{y}^{+}=10.9, \Delta \mathrm{z}^{+}$ $=0.70 \sim 30.5$. The mesh is uniform in the streamwise and spanwise directions and non-uniform in the vertical direction. $R e_{z i}\left(=U_{m} z_{i} / v\right), \operatorname{Re\tau }\left(=u_{*} \delta / v\right), R i_{b}\left(=\left(g / \theta_{o}\right)\left(\theta_{m}-\theta_{s}\right) z_{i} / U_{m}{ }^{2}\right)$ and $R i_{t}\left(=\left(g / \theta_{t}\right)(\Delta \theta)_{t} z_{i} / w_{*}{ }^{2}\right)$ are about $9000,333,-0.50$ and 22.4 , respectively. Here, $U_{m}, v$, $\mathrm{u}_{*}, \mathrm{~g}, \theta_{t}$ and $\Delta \theta_{t}$ are wind velocity which remains constant with height inside the CBL, kinematic viscosity, friction velocity, gravity acceleration, averaged absolute temperatures within the inversion layer and the temperature increment across the inversion, respectively. Table1 shows the numerical conditions.

\begin{tabular}{|l|l|}
\hline Region size $(\mathrm{Lx} \times \mathrm{Ly} \times \mathrm{Lz})$ & $40.0 \delta \mathrm{o} \times 6.4 \delta \mathrm{o} \times 3.0 \delta \mathrm{o}$ \\
\hline Grid number $(\mathrm{Nx} \times \mathrm{Ny} \times \mathrm{Nz})$ & $400 \times 128 \times 90$ \\
\hline Resolution $\left(\triangle \mathrm{x}^{+} \times \Delta \mathrm{y}^{+} \times \Delta \mathrm{z}^{+}\right)$ & $22.0 \times 10.9 \times 0.70 \sim 30.5$ \\
\hline $\mathrm{Re}_{\mathrm{zi}}, \operatorname{Re} \tau$ & 9000,333 \\
\hline $\mathrm{Ri}_{\mathrm{b}}, \mathrm{Ri}_{\mathrm{t}}$ & $-0.50,22.4$ \\
\hline
\end{tabular}

Table1 Numerical conditions

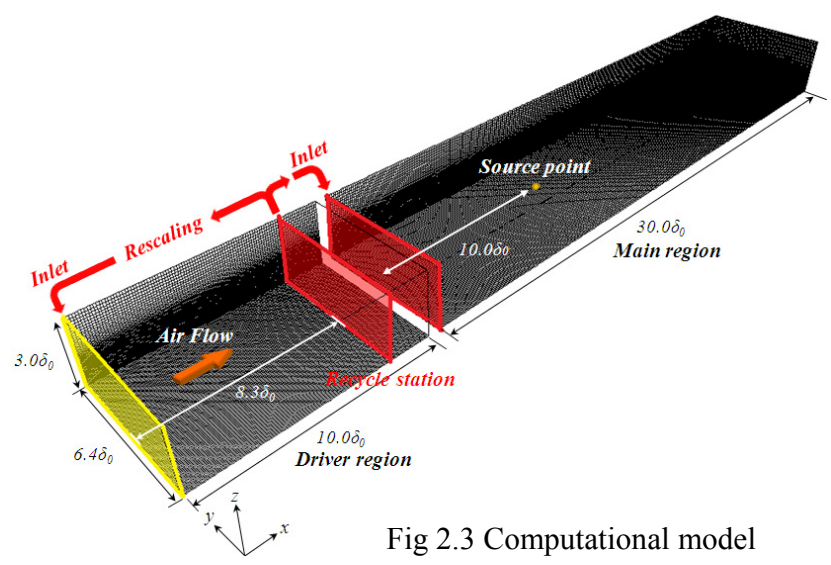

\subsection{Governing Equations}

In this study, LES is performed using dynamic Smagorinsky models for flow and temperature and dispersion fields. The incompressible Navier-Stokes, scalar conservation (temperature and concentration) and continuity equations are represented by the following equations.

$$
\begin{aligned}
& \frac{\partial \bar{u}_{j}}{\partial x_{j}}=0 \\
& \frac{\partial \bar{u}_{i}}{\partial t}+\bar{u}_{j} \frac{\partial \bar{u}_{i}}{\partial x_{j}}=-\frac{1}{\rho} \frac{\partial \bar{p}}{\partial x_{i}}+\frac{\partial}{\partial x_{j}} v\left(\frac{\partial \bar{u}_{i}}{\partial x_{j}}+\frac{\partial \bar{u}_{j}}{\partial x_{i}}\right)-\frac{\partial}{\partial x_{j}} \tau_{i j}+g \beta \bar{\theta} \delta_{i 2} \\
& \frac{\partial \bar{\theta}}{\partial t}+\bar{u}_{j} \frac{\partial \bar{\theta}}{\partial x_{j}}=\frac{\partial}{\partial x_{j}}\left(\frac{v}{\operatorname{Pr}} \frac{\partial \bar{\theta}}{\partial x_{j}}\right)-\frac{\partial}{\partial x_{j}} h_{j} \\
& \frac{\partial \bar{c}}{\partial t}+\bar{u}_{j} \frac{\partial \bar{c}}{\partial x_{j}}=\frac{\partial}{\partial x_{j}}\left(\frac{v}{S c} \frac{\partial \bar{c}}{\partial x_{j}}\right)-\frac{\partial}{\partial x_{j}} S_{j} \\
& \tau_{i j}=\overline{u_{i} u_{j}}-\bar{u}_{i} \bar{u}_{j} \\
& h_{j}=\overline{u_{j} \theta}-\bar{u}_{j} \bar{\theta}
\end{aligned}
$$




$$
\begin{aligned}
& s_{j}=\overline{u_{j} c}-\bar{u}_{j} \bar{c} \\
& \tau_{i j}-\frac{1}{3} \delta_{i j} \tau_{k k}=-v_{S G S}\left(\frac{\partial \bar{u}_{i}}{\partial x_{j}}+\frac{\partial \bar{u}_{j}}{\partial x_{i}}\right) v_{S G S}=C \overline{\Delta^{2}}|\bar{S}| \\
& h_{j}=-\frac{v_{S G S}}{P r_{S G S}} \frac{\partial \bar{\theta}}{\partial x_{j}} \\
& s_{j}=-\frac{v_{S G S}}{S c_{S G S}} \frac{\partial \bar{c}}{\partial x_{j}}
\end{aligned}
$$

where $u_{i}, \theta, c, t, \rho, p$ and $\beta$ are velocity, temperature, concentration, time, density, pressure and thermal expansion coefficient, respectively. $i$ stands for coordinates $(1,2,3$ : streamwise, spanwise, vertical). $R e, P r$ and $S c$ are Reynolds number, Prandtl number and Schmidt number. $\tau_{i j}, h_{j}$ and $s_{j}$ are the subgrid-scale Reynolds stress, heat flux and scalar flux. Here, the turbulent Schmidt number $S c_{s g s}$ is set to a constant value of 0.5 .

\subsection{Numerical Discretization and Algorithm}

The coupling algorithm of the velocity and pressure fields is based on a fractional-step method. The convection and diffusion terms in the flow field are discretized by the Adams-Bashforth and the Crank-Nicolson schemes, respectively, for time integration. The convection and diffusion terms in the temperature and concentration fields are discretized by the Adams-Bashforth scheme for time integration. The Poisson equation is solved by SOR method. For the spatial discretization in the governing equation, a second-order accurate central difference is used. However, for the concentration field, a third-order upwind scheme is used for the convection term only around a point source.

\section{Validation of Characteristics of Inflow Turbulence and Plume Dispersion -Comparison with Neutral Boundary Layer Case ${ }^{[4]}$}

In this study, we perform LES on atmospheric dispersion under neutral conditions and validate the numerical model in order to extend the LES model to dispersion in the CBL flow.

Figure 3.1 compares the vertical profiles of mean velocity $\left(\mathrm{u}_{\text {mean }}\right)$ at the source location and each component of the turbulent intensities at the inlet of the main region(LES_urms, vrms, wrms(Inlet)) and the source location(LES_urms, vrms, wrms(SourcePoint)) in the simulated neutral boundary layer with the experimental data ${ }^{[4]}$. The source is located at a distance of $10 \delta_{\mathrm{o}}$ downwind of the inlet of the main region and elevated $0.19 \delta(\delta$ : thickness of NBL at inlet of main region) from the ground surface. The mean velocity and each component of the turbulent intensities are normalized by a free stream velocity $\left(U_{\infty}\right)$. The LES results for the mean velocity profile at the source location are in good agreement with experimental ${ }^{[4]}$ results. It is found that the LES results for each component of the turbulent intensities at the source location show good agreement with experimental results at $\mathrm{z} / \delta>0.2$. However, vertical component of the turbulent intensities obtained by LES are smaller than the experimental results conducted by Fackrell et al. ${ }^{[4]}$ near the ground surface. Here, focusing on the turbulent intensities profiles at the inlet of the main region, we can find that the LES data are similar to the wind tunnel experimental data conducted by Degraaff et al. ${ }^{[13]}$. This indicates that the turbulent fluctuations in the spatially-developing boundary layer flow are successfully generated using the rescaling method ${ }^{[11]}$.

Figure 3.2 compares the vertical profiles of mean $\left(\mathrm{c}_{\text {mean }}\right)$ and r.m.s $\left(\mathrm{c}_{\mathrm{rms}}\right)$ concentrations with the experimental data ${ }^{[4]}$. The vertical coordinate is normalized by the source height 
(Hs). The mean and r.m.s concentrations are normalized by the maximum mean $\left(\mathrm{c}_{\text {mean_max }}\right)$ and r.m.s ( $\left.c_{\text {rms_max }}\right)$ concentrations at each distance, respectively. The LES results for the vertical profiles of mean and r.m.s concentrations show good agreement with the experimental data ${ }^{[4]}$ at each distance.

This validates the LES model for plume dispersion in NBL flow using Lund's method ${ }^{[11]}$ and we extend this LES technique to plume dispersion in the CBL flow.
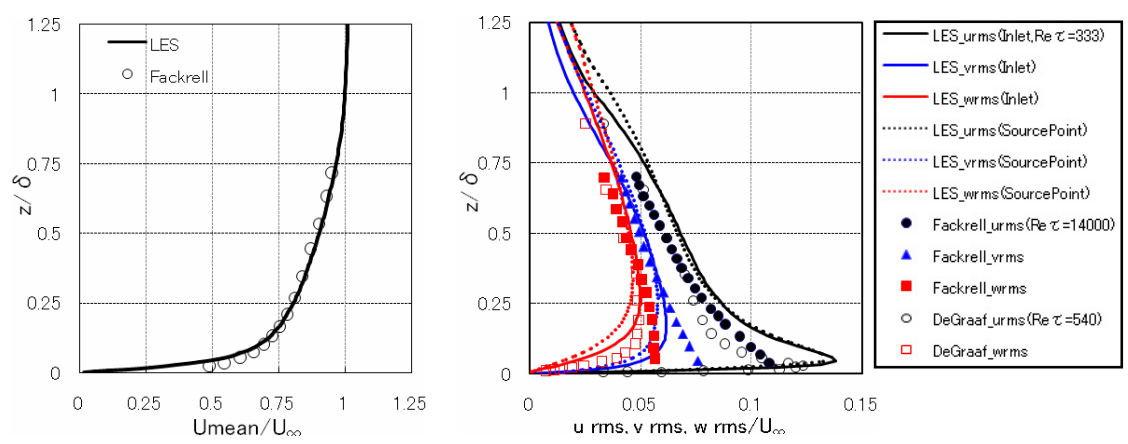

Figure 3.1 Vertical profiles of mean velocity and turbulent intensity
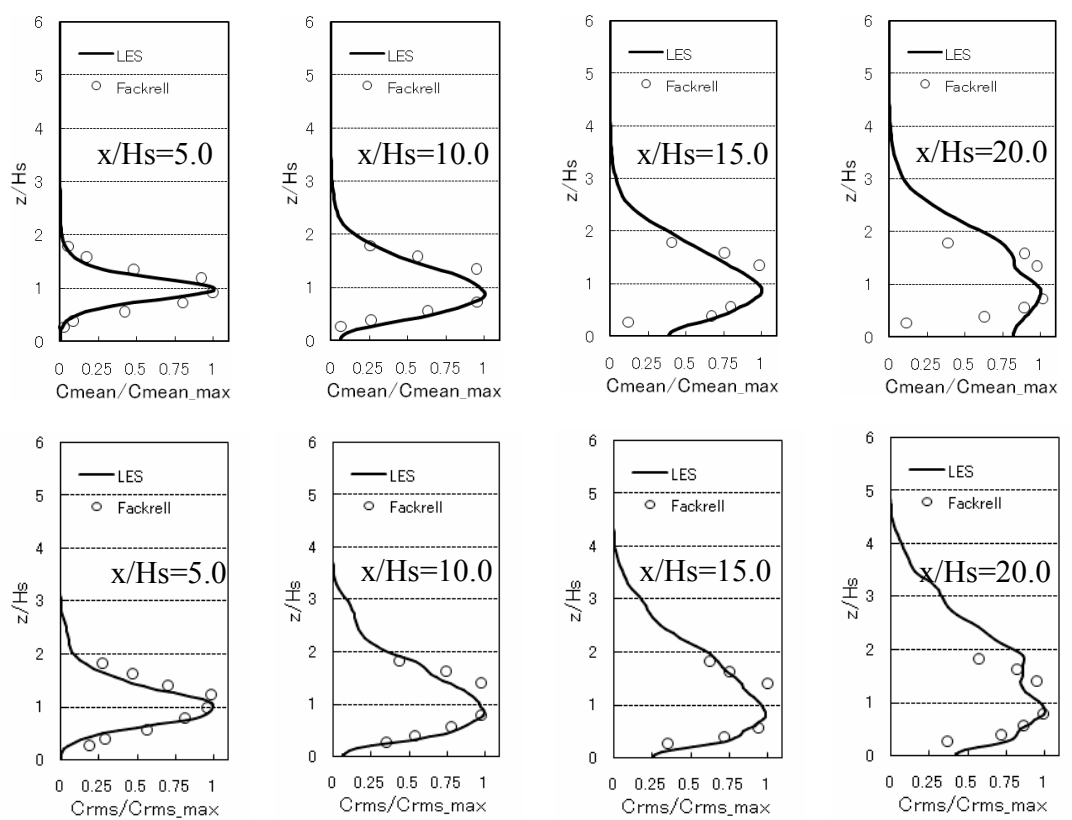

Figure 3.2 Vertical profiles of mean and r.m.s concentrations

\section{Computational Results for Flow and Temperature Fields in the CBL Flow -Comparison with Experimental ${ }^{[2]}$ and Observed ${ }^{[3]}$ Data-}

Figure 4.1 shows a shot of an instantaneous temperature field in the CBL flow. It is clearly found that the vertical temperature distribution becomes homogeneous and the vertical turbulent motions across the inversion become more active with downwind distance.

Figures 4.2, 4.3 compare streamwise variation of mean velocity and temperature profiles obtained by LES with experimental data ${ }^{[2]}$. Mean velocity and temperature are normalized by wind velocity $\left(U_{m}\right)$ and temperature in the range where values are constant with height inside the CBL, respectively. Here, Hs stands for the source height. In particular, at larger distances from the inlet of the main region $(\mathrm{x} / \mathrm{Hs}=56.2)$, mean velocity and temperature profiles obtained by LES are constant in the range from $0.2 z_{i}$ to $0.8 z_{i}$. However, mean 
velocity and temperature profiles obtained by a wind tunnel experiment ${ }^{[2]}$ are constant in the range from $0.2 z_{i}$ to $0.6 z_{i}$. For LES data, there is an underprediction around the inversion height. However, the characteristics obtained by LES, such as uniform distributions of mean velocity and temperature in the middle portion of the $\operatorname{CBL}\left(\mathrm{z} / \mathrm{z}_{\mathrm{i}}=0.2 \sim 0.8\right)$ are similar to the previous experimental data $^{[2]}$. Furthermore, the characteristics obtained by LES, such as increments of wind speed and temperature in the upper portion of the $\operatorname{CBL}\left(\mathrm{z} / \mathrm{z}_{\mathrm{i}}=0.8 \sim\right)$ also present similar results for the previous experimental data ${ }^{[2]}$.

Figure 4.4 compares streamwise variation of vertical velocity variances $\left(\mathrm{w}^{2}\right)$ profiles obtained by LES with experimental data ${ }^{[2]}$. Vertical velocity variances are normalized by the convective velocity scale $\left(\mathrm{w}_{*}\right)$. Vertical velocity variances obtained by LES show good agreement with the experimental ${ }^{[2]}$ and observed data ${ }^{[3]}$ except near the ground surface at each distance. Figure 4.5 compares streamwise variation of temperature variances $\left(\theta^{2}\right)$ profiles obtained by LES with experimental data ${ }^{[2]}$. Temperature variances are normalized by the convective temperature $\operatorname{scale}\left(\theta_{*}\right)$. LES data for the positions of both maximum temperature variances in the inversion layer and the minimum temperature variances inside the CBL are a little higher than the experimental ${ }^{[2]}$ observed $^{[3]}$ data. However, it is clearly found that LES data are qualitatively in good agreement with the experimental ${ }^{[2]}$ and observed $^{[3]}$ data, and the main features of the present LES model, such as the distribution patterns of the vertical velocity and temperature variances throughout the CBL are similar to those data $^{[2],[3]}$.

Figures 4.6, 4.7 compare streamwise variation of streamwise and vertical heat flux profiles, respectively, obtained by LES with experimental data ${ }^{[2]}$. The streamwise and vertical heat fluxes are normalized by the friction velocity $\left(\mathrm{u}_{*}\right)$, the convective velocity scale and the convective temperature scale. At larger distances $(\mathrm{x} / \mathrm{Hs}=56.2)$, especially, maximum streamwise heat fluxes near the inversion are overestimated. Vertical heat fluxes obtained by LES are quantitatively different from experimental data ${ }^{[2]}$ inside the CBL. However, the characteristics, such as minimum streamwise heat fluxes near the ground surface and increment towards the inversion height, maximum vertical heat fluxes near the ground surface and decrement towards the inversion height, are similar to the experimental data ${ }^{[2]}$. As mentioned above, the characteristics such as uniform distributions of mean velocity and temperature in the main portion of CBL flow, the pattern of the streamwise heat flux distribution and decrement of vertical heat flux towards the inversion height are similar to those of previous experiments ${ }^{[1],[2]}$ and observations ${ }^{[3]}$. The Richardson number based on the temperature increment across the inversion obtained by the present LES model is 22.4. This value is the same order as the previous wind tunnel experiments ${ }^{[1],[2]}$. This indicates that the capping inversion strength in the present LES model is successfully simulated.

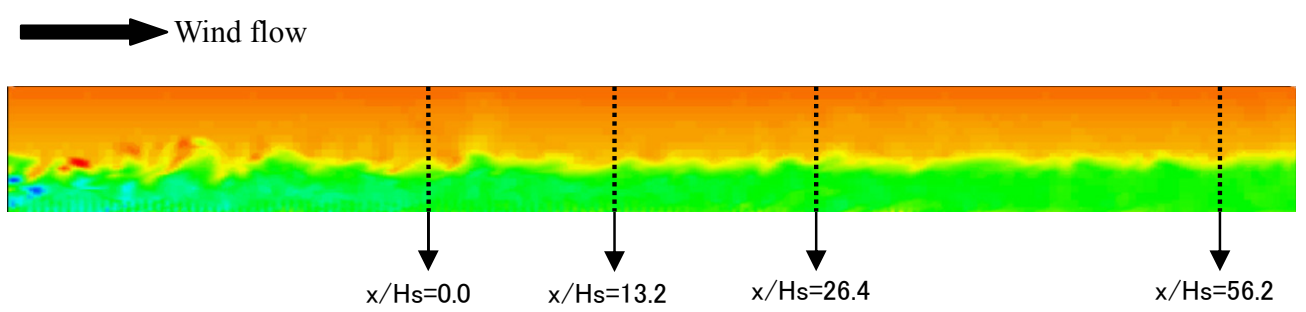

Figure 4.1 Instantaneous temperature field in main region 

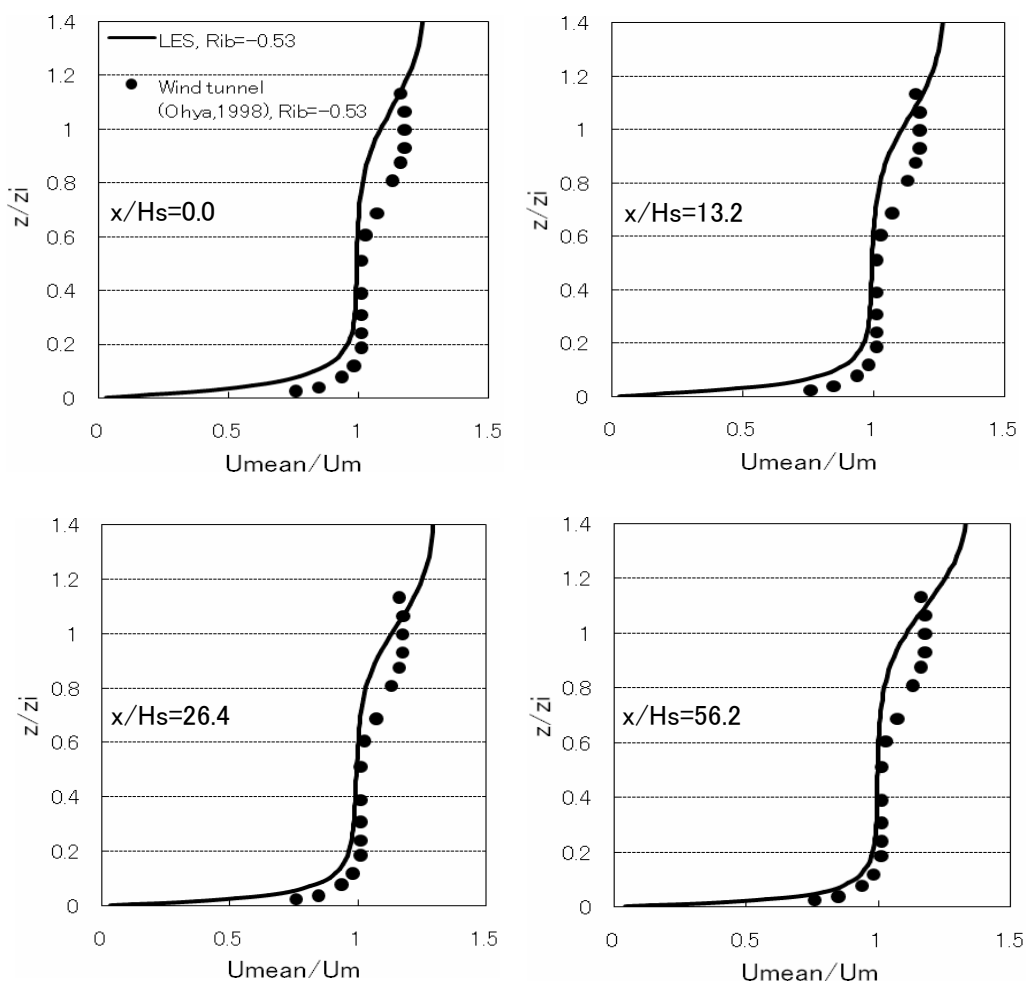

Figure 4.2 Streamwise variation of mean velocity profiles
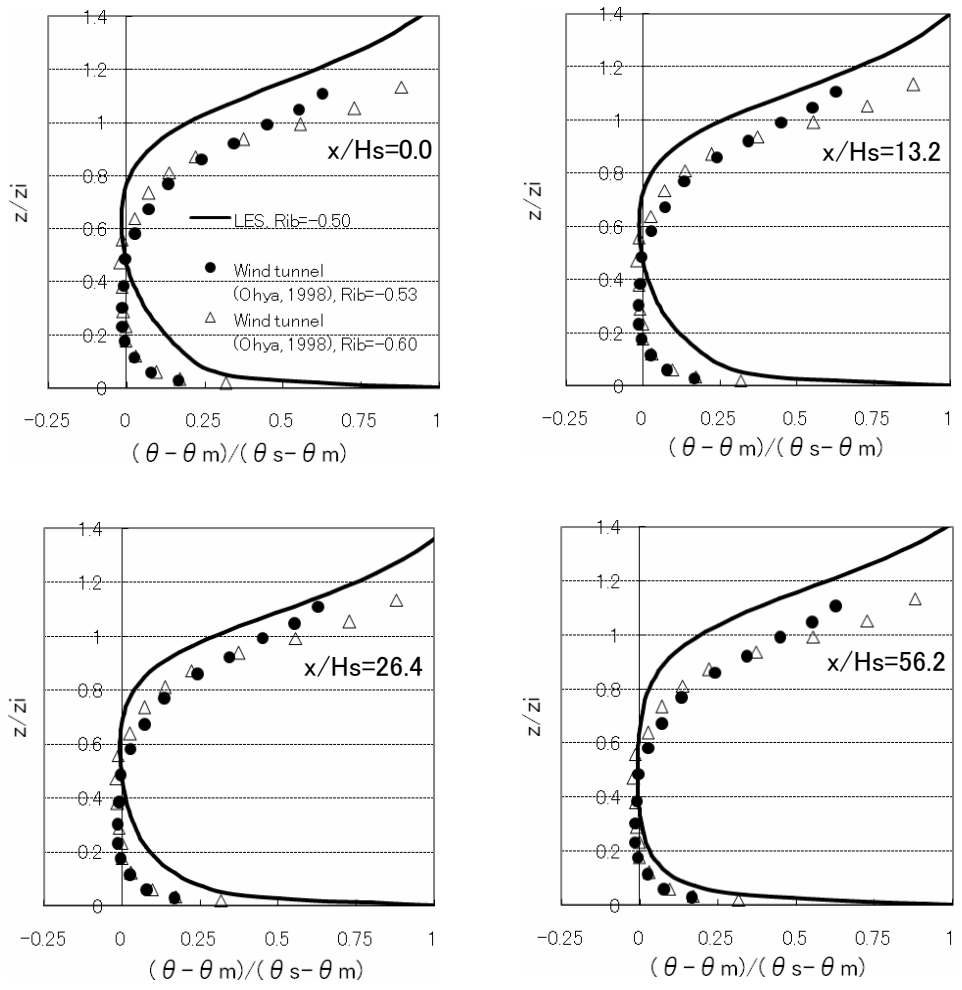

Figure 4.3 Streamwise variation of mean temperature profiles 

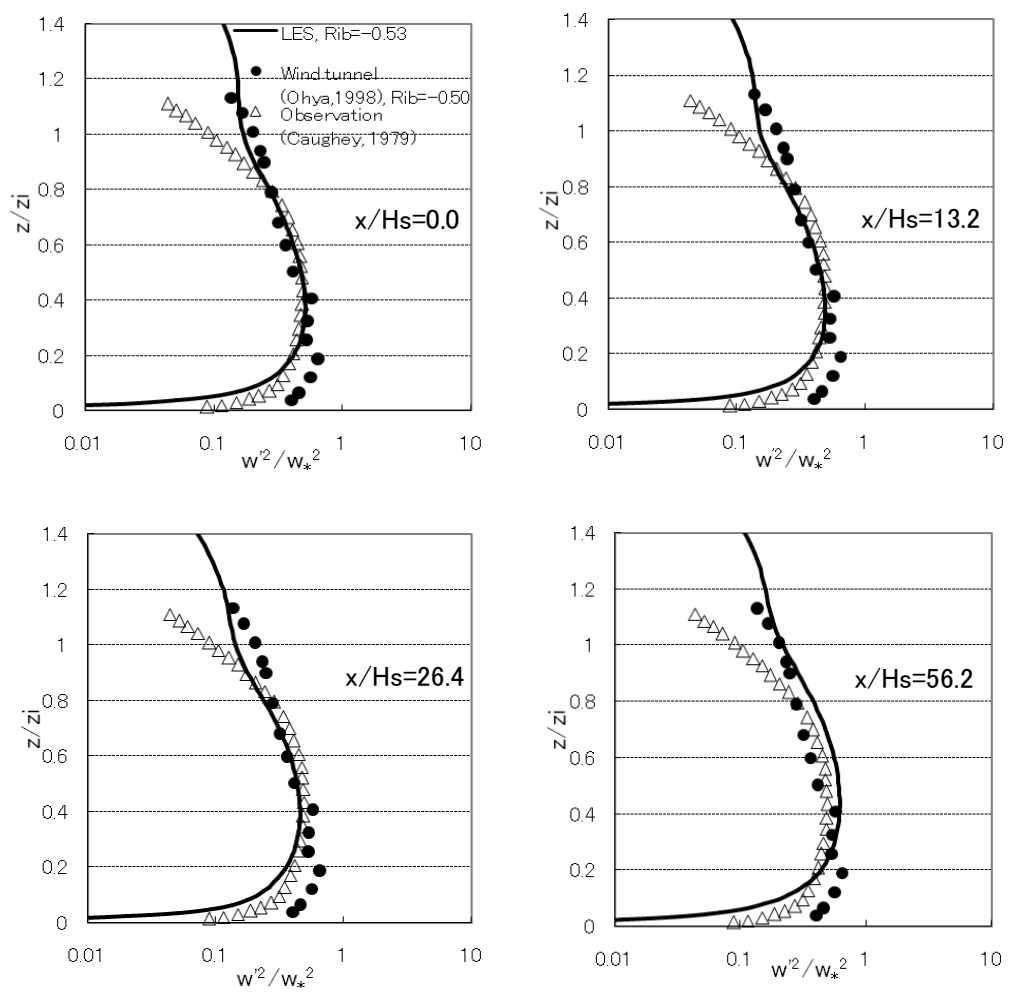

Figure 4.4 Streamwise variation of vertical velocity variance profiles
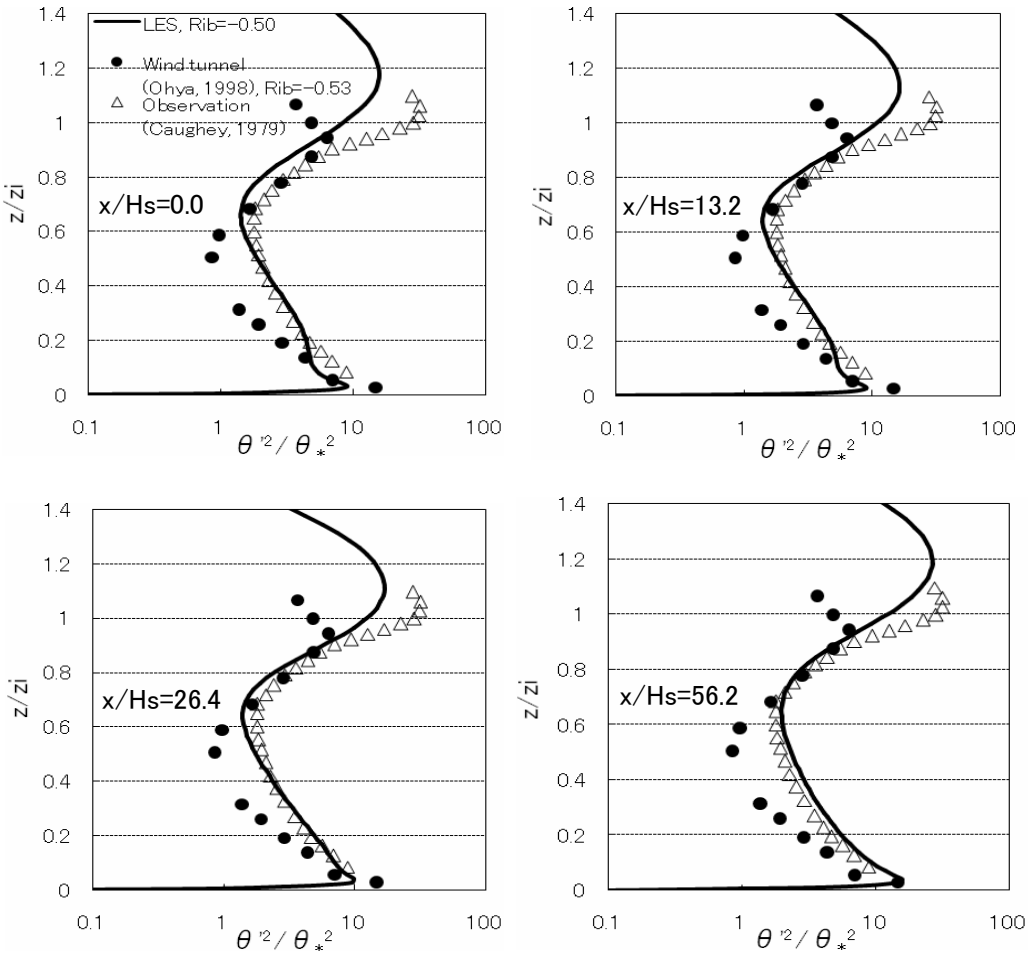

Figure 4.5 Streamwise variation of temperature variance profiles 

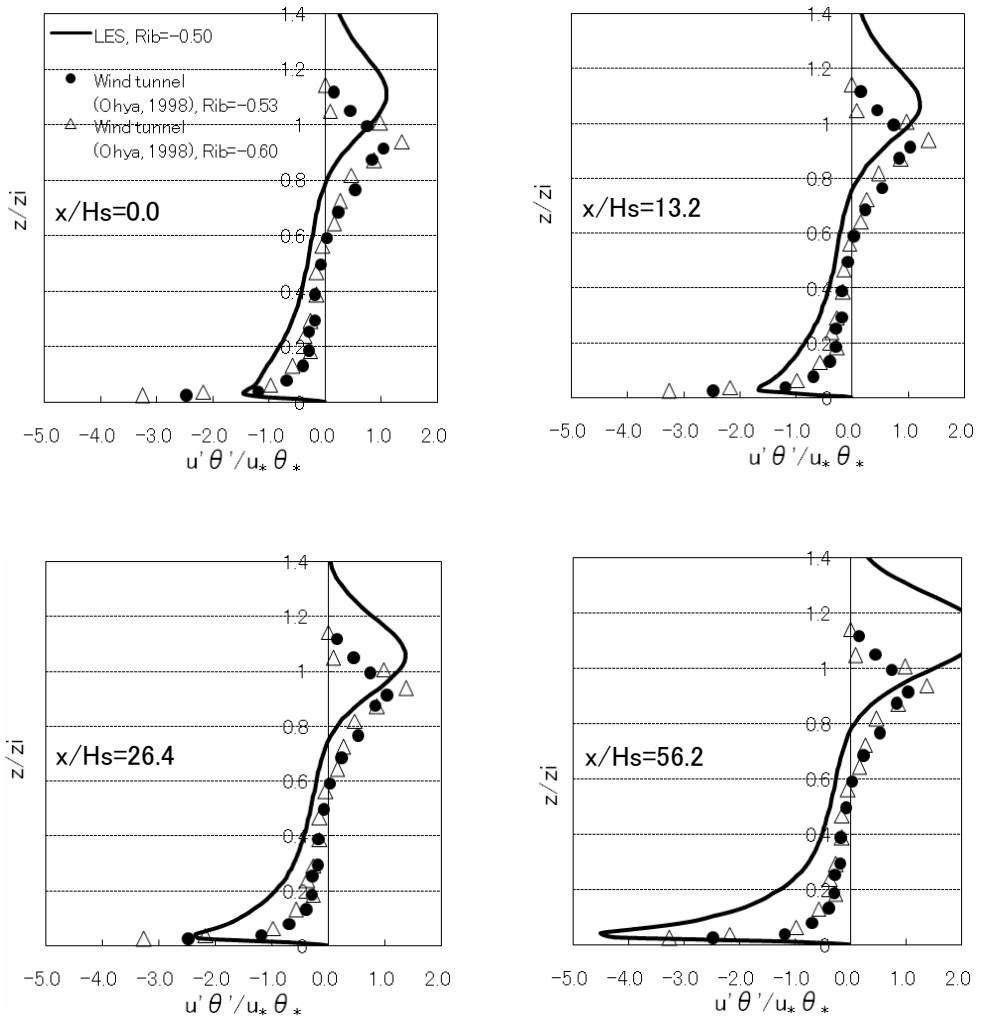

Figure 4.6 Streamwise variation of streamwise heat flux profiles
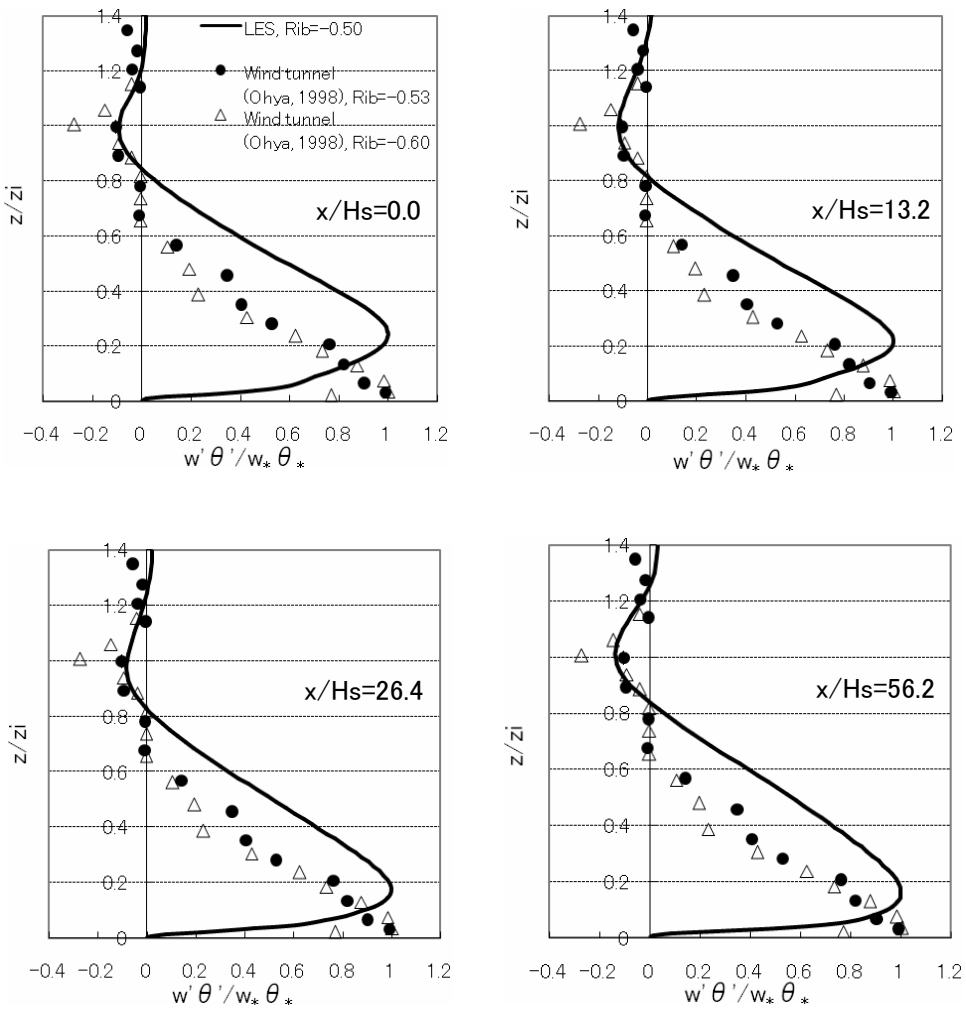

Figure 4.7 Streamwise variation of vertical heat flux profiles 


\section{Computational Results for Dispersion Field in the CBL Flow}

Figure 5.1 shows a shot of an instantaneous concentration field in NBL and CBL flows obtained by LES. In the NBL flow, the vertical spread of the plume increases with downwind distance. In the CBL flow, however, high concentration regions of the plume touch down at a shorter distance from the point source. The vertical spread of the plume in the CBL flow is larger than that in the NBL flow due to the active vertical turbulent motions.

Figure 5.2 shows the streamwise variation of mean $\left(\mathrm{c}_{\text {mean }}\right)$ concentrations profiles obtained by LES. The point source height is set to $0.27 \mathrm{z}_{\mathrm{i}}$ which corresponds to the source height of $0.19 \delta$. Mean concentration is normalized by velocity of effluent (Ue), the source height and the source concentration (Co). Here, the source concentration means the concentration measured at nominal zero time. In the NBL flow, the vertical spread of the plume increases with downwind distance from the point source. Therefore, mean concentration values near the ground surface become larger at each distance. These patterns of concentration distribution are similar to those obtained by the previous wind tunnel experiment ${ }^{[4]}$. In the CBL flow, due to the enhanced vertical plume spread, mean concentrations at shorter distances from a point source $(\mathrm{x} / \mathrm{Hs}=7.6,13.2)$ are larger than those of the NBL case near the ground surface. Furthermore, the peak positions of mean concentration are moved upwards with downwind distance. However, at much larger distances from the point sources $(\mathrm{x} / \mathrm{Hs}=56.2)$, the vertical plume spread is restricted by the blocking effect of the capping inversion and the mean concentrations decrease immediately above the inversion height. This plume behavior in the CBL flow is also similar to that in the previous wind tunnel ${ }^{[1]}$ and water tank experiments ${ }^{[14]}$.

Figure 5.3 shows streamwise variation of r.m.s concentration $\left(\mathrm{c}_{\mathrm{rms}}\right)$ profiles obtained by LES. R.m.s concentration is also normalized by velocity of effluent, the source height and the source concentration. In the NBL flow, the peak positions of r.m.s concentrations are moved upwards with downwind distance. These results are the same as in the previous wind tunnel experiment ${ }^{[1]}$. In the CBL flow, vertical spread of r.m.s concentrations is rapid due to active vertical turbulent transport and especially at larger distances from the point source $(\mathrm{x} / \mathrm{Hs}=26.4$, 56.2), r.m.s concentration profiles become uniform inside the CBL. Furthermore, it is found that the peak position of r.m.s concentration rises towards the inversion height at $\mathrm{x} / \mathrm{Hs}=26.4$ and are maintained even at much larger distances from the point source $(\mathrm{x} / \mathrm{Hs}=56.2)$. Above the capping inversion, r.m.s concentrations also decrease rapidly.

(a)NBL

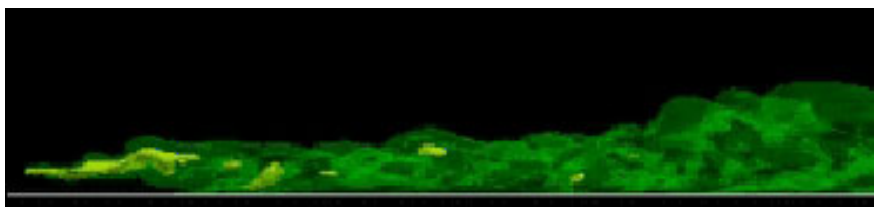

(b) $\mathrm{CBL}$

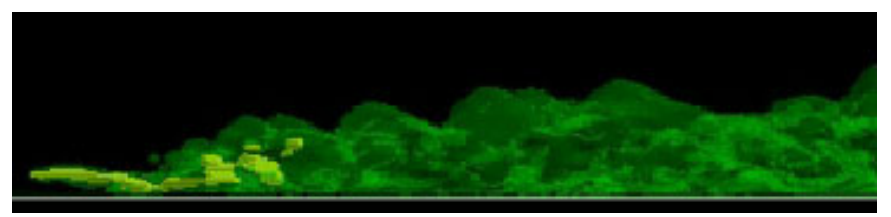

$1 \%$ of initial concentration $0.01 \%$ of initial concentration

Figure 5.1 Instantaneous concentration field in (a)NBL and (b)CBL flows 
Figure 5.4 shows vertical profiles of vertical concentration fluxes (w'c') obtained by LES at specified distances from the point source. Vertical concentration fluxes are normalized by velocity of effluent and the source concentration. In the NBL flow, at shorter distances $(\mathrm{x} / \mathrm{Hs}=7.6,13.2)$, the shape of the flux profiles is antisymmetric at the source height. At larger distances $(\mathrm{x} / \mathrm{Hs}=26.4,56.2)$, the vertical concentration flux profiles become almost uniform. In the CBL flow, at much shorter distances from the point source $(\mathrm{x} / \mathrm{Hs}=7.6)$, the shape of the vertical concentration flux profiles is antisymmetric at the source height as in the NBL case. Furthermore, the absolute peak values of the vertical concentration flux above the source height are much larger than those below the source height. With downwind distance $(\mathrm{x} / \mathrm{Hs}=7.6,13.2,26.4)$, the peak positions of the positive vertical concentration flux above the source height are moved upwards. However, a capping inversion blocks the vertical turbulent transport of the plume and the vertical concentration flux values decrease towards the capping inversion. However, the profiles of the vertical concentration flux below the source height become uniform. At much larger distances from the point source $(\mathrm{x} / \mathrm{Hs}=56.2)$, the vertical concentration flux profiles become uniform throughout the CBL.

As discussed above, in the CBL flow, the plume is rapidly dispersed in the vertical direction due to the active turbulent motions. R.m.s concentrations are distributed more widely in the vertical direction than in the NBL flow case even at shorter distances from the point source. With downwind distance, the plume becomes mixed throughout the CBL. However, the upward spread of the plume is restricted due to the capping effects of the temperature inversion. As a result, the mean concentration values decrease rapidly towards the inversion height. This large concentration gradient around the inversion height produces the fluctuating of concentration. This characteristic is maintained even at much larger distances from the point source and the peak positions of r.m.s concentration are located near the inversion height.
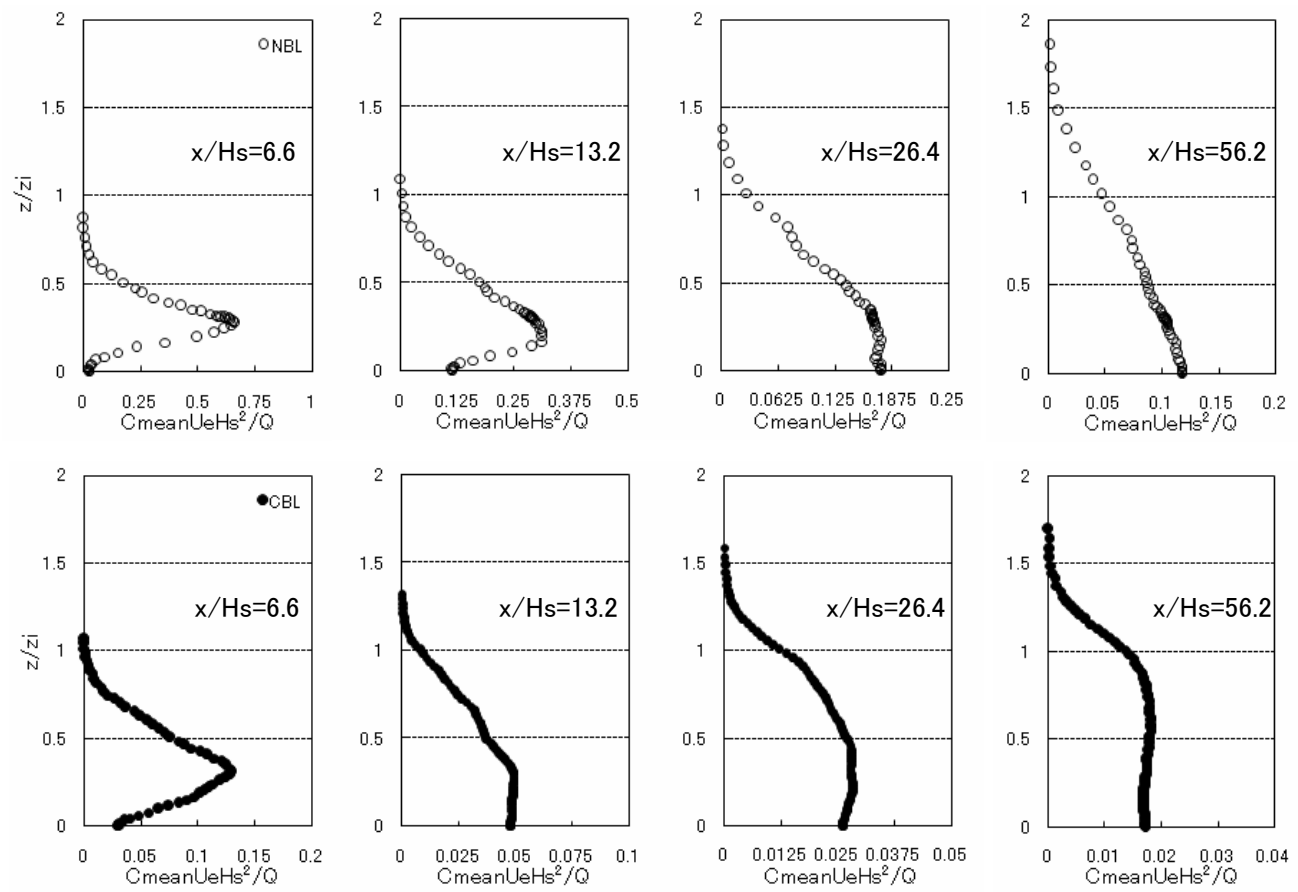

Figure 5.2 Vertical profiles of mean concentration in NBL and CBL flows 

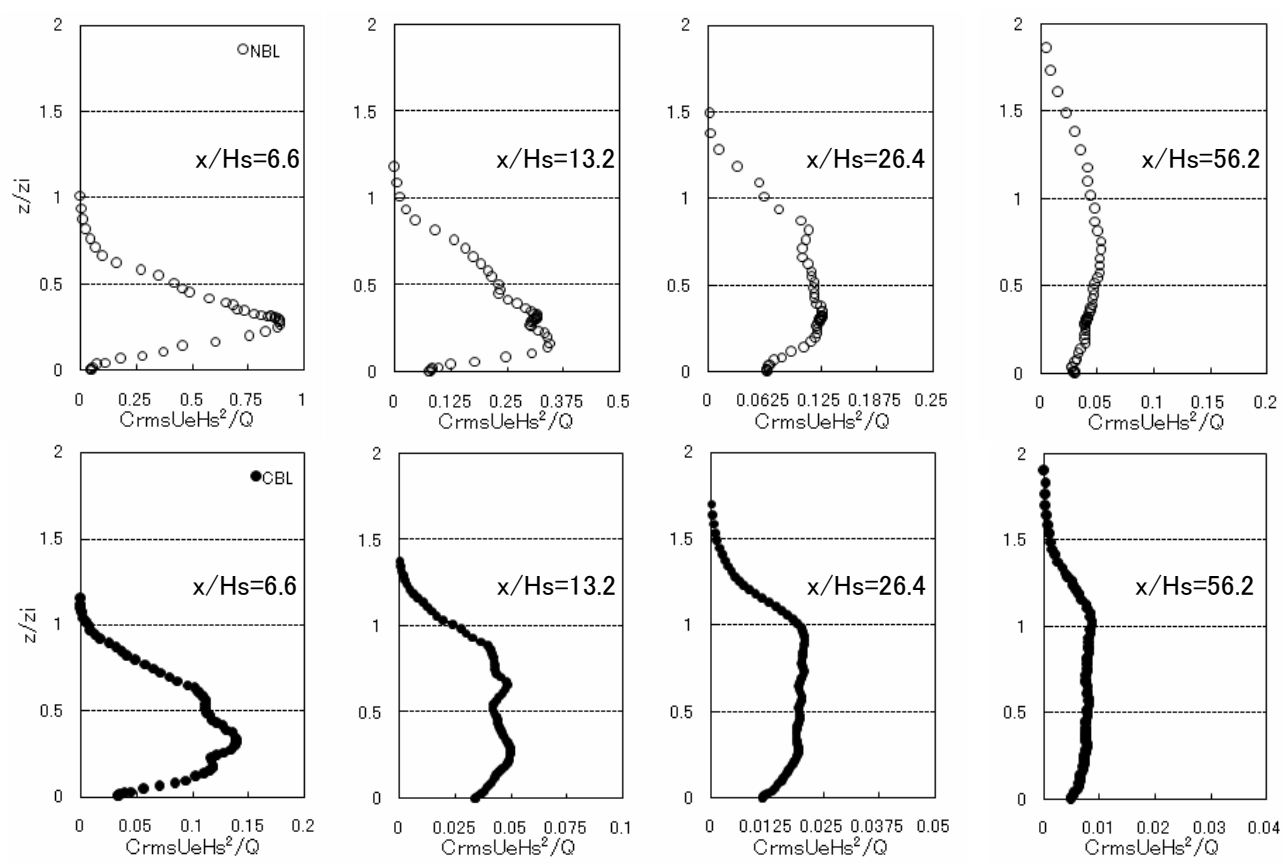

Figure 5.3 Vertical profiles of r.m.s concentration in NBL and CBL flows

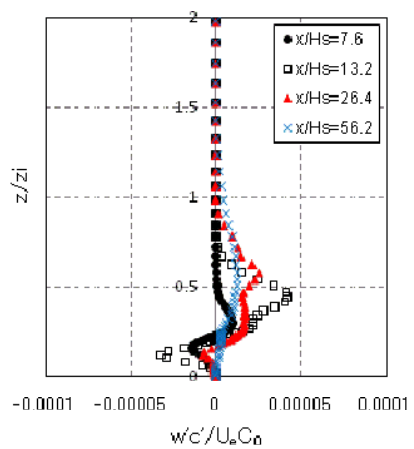

(a) NBL case

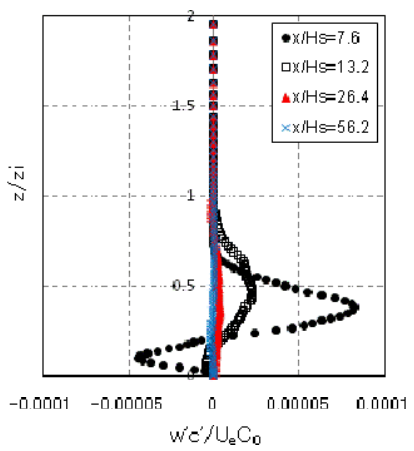

(b) CBL case

Figure 5.4 Vertical profiles of vertical concentration flux

\section{Conclusion}

We have proposed the LES model for atmospheric dispersion in a spatially-developing CBL flow capped by a temperature inversion. This paper has compared plume behavior in the CBL flow with that in the NBL flow and studied the capping effects of temperature inversion on the characteristics of mean and fluctuating concentrations. Obtained results are as follows:

(1)We validate the LES model for spatially-developing CBL flow capped by a temperature inversion using Lund's method. The Richardson number based on the temperature increment across the inversion obtained by the present LES model is the same order as the previous wind tunnel experiments ${ }^{[6],[9]}$ and the capping effect of the temperature inversion can be captured qualitatively in the upper portion of the CBL. The characteristics such as uniform distributions of mean velocity and temperature in the main portion of CBL flow, the pattern of the streamwise heat flux distribution and decrement of vertical heat flux towards the inversion height are similar to those of previous experiments ${ }^{[6],[9]}$ and observations ${ }^{[10]}$. Thus, we have successfully simulated the spatially-developing CBL flow capped by a temperature inversion. 
(2)We apply the LES model to atmospheric dispersion in a simulated spatially-developing CBL flow capped by a temperature inversion. The vertical spread of a plume in CBL flow is much larger than in NBL flow even at shorter distances from the point source. At larger distances from the point source, the upward spread of the plume begins to be influenced by the capping inversion, and mean concentration values decrease rapidly towards the inversion height. It is found that the capping effect of temperature inversion can be captured qualitatively in the upper portion of the CBL.

(3)At shorter distances from the point source, the vertical profiles of r.m.s concentration are distributed more widely than those in the NBL flow. At larger distances from the point source, the skewed distribution of mean concentration near the inversion height produces a fluctuating concentration in the upper portion of the CBL. This characteristic is maintained even at much larger distances from the point source and the peak positions of r.m.s concentration are located near the inversion height.

\section{References}

(1) Fedorovich.E., Thater.J., A wind tunnel study of gaseous tracer dispersion in the convective boundary layer capped by a temperature inversion, Atmospheric Environment, 36, 2245-2255, 2002.

(2) Ohya,Y., Uchida,T., Laboratory and numerical studies of the convective boundary layer capped by a strong inversion, Boundary Layer Meteorology, 112, 223-240, 2004.

(3) Caughey,S.J., S.G.Palmer, Some aspects of turbulence structure through the depth of the convective boundary layer, Quarterly Journal of the Royal Meteorological Society, 105, 811-827, 1979.

(4) Fackrell.J.E., Robins.A.G., Concentration fluctuation and fluxes in plumes from point sources in a turbulent boundary layer, Journal of Fluid Mechanics, 117, 1-26, 1982.

(5) Sada, K., Sato, A., Wind tunnel experiment of tracer gas concentration fluctuation in turbulent boundary layer: characteristics of concentration fluctuation with low concentration threshold, Journal of the Japan Society of Atmospheric Environment, 34-5, 337-351, 1999a (in Japanese).

(6) Sada, K., Sato, A., Wind tunnel experiment of tracer gas concentration fluctuation in atmosphere: similarity of concentration fluctuation in plume from elevated source, Transactions of the Japan Society of Mechanical Engineers, 65-636, 2734-2742, 1999b (in Japanese).

(7) Sada, K., Wind tunnel experiment of tracer gas diffusion within unstable boundary layer over coastal region, Atmospheric Environment, 36, 4757-4766, 2002.

(8) Sada, K., Wind tunnel experiment on flow and tracer gas diffusion in convective planetary boundary layer, JSME International Journal, 39B, 1, 19-27, 1996.

(9) Spalart, P. R. and Watmuff, J. H., Experimental and numerical study of a turbulent boundary layer with pressure gradients, Journal of Fluid Mechanics, 249, 337,1993.

(10) Maeder, T., Adams, N. A. and Kleiser, L., Direct simulation of turbulent supersonic boundary layers by an extended temporal approach, Journal of Fluid Mechanics, $429,187,2001$.

(11) Lund,T.S., Wu,X., Squires,K.D., Generation of Turbulent Inflow Data for Spatially Developing Boundary Layer Simulations, Journal of Computer Physics, 140, 233-258,1998.

(12) Tamura,T., Furusawa, T. and Mori, K.: DNS/LES of spatially-developing thermally stratified turbulent boundary layers, Proceedings of the 18th national symposium on wind engineering, 51-56, 2004.

(13) Degraaff, D. B. and Eaton, J. K., Reynolds-number Scaling of the Flat-plate Turbulent Boundary Layer, Journal of Fluid Mechanics, 422, 319-346,2000.

(14) Willis,G.E., Deardorff,J.W., Buoyant plume dispersion and inversion entrapment in and above a laboratory mixed layer, Atmospheric Environment, 21, 1725-1735, 1987. 